\title{
ANÁLISIS DEL CAMBIO TEMPORAL Y ESPACIAL DEL USO DEL SUELO EN LA REGIÓN CENTRO-SUR DE CHILE
}

\author{
TEMPORAL AND SPATIAL ANALYSIS OF LAND USE CHANGE IN THE SOUTH CENTRAL RE- \\ GION OF CHILE
}

\author{
Gastón Vergara Díaz Víctor Sandoval Vázquez $^{2} \quad$ Miguel Ángel Herrera Machuca $^{3}$
}

\begin{abstract}
RESUMEN
La investigación estudia el cambio temporal y espacial del uso del suelo en la Región del Bio- Bio (Centro-Sur de Chile), para el periodo 1998 y 2008. Se utilizó la cobertura digital del monitoreo nacional del año 1998 y fotografías aéreas para el año 2008, posteriormente se construyeron matrices que explican el cambio $\mathrm{y}$ transiciones entre los diferentes usos del territorio. Las superficies con plantaciones crecieron a una tasa anual de $2,5 \%$, incremento generado por el consumo de praderas y matorrales en $21 \%$, terrenos agrícolas $14 \%$, bosque mixto $8,8 \%$, humedales en $1,6 \%$ y bosque nativo en menor medida $1,1 \%$. Las praderas y matorrales presentaron una pérdida a una tasa de $2,5 \%$ anual, la causa principal se debe a la transferencia de este uso a plantaciones con un $21 \%$ y a otros usos como terrenos agrícolas $2 \%$. Los terrenos agrícolas perdieron superficie con una tasa anual de $1,5 \%$, terrenos que incrementaron a las plantaciones en $14 \%$, los humedales perdieron superficie con tasa de $0,4 \%$, la superficie se trasladó a las áreas urbanas e industriales en 2,6 \% y plantaciones en 1,6\%. La principal presión para ocupar superficie, corresponde a las plantaciones, debido a que la Región del Bio-Bio representa la mayor cantidad de superficie (858.592 ha) de plantaciones exóticas del país.
\end{abstract}

Palabras clave: monitoreo; territorio; plantaciones.

\begin{abstract}
This research examines the spatial and temporal changes of the land use in the Bio-Bio Region (CentralSouth of Chile), for the years 1998 and 2008. We used the national monitoring digital coverage of 1998 and aerial photographs of 2008. Subsequently, we constructed matrices that explain the changes and transitions between different land uses. Plantations grew at an annual rate of $2.5 \%$ due to grassland and shrub consumption in $21 \%$, agricultural land 14\%, in mixed forest it grew by $8.8 \%$ annually, while in wetlands it grew by $1.6 \%$ and native forest had the least growth rate of $1.1 \%$. Grasslands and shrub lands showed a loss at a rate of $2.5 \%$ per year caused mainly by their conversion into plantations at $21 \%$ and to other agricultural land uses at $2 \%$. Agricultural land surface was lost at an annual rate of $1.5 \%$. Land plantations had a percentage increase of $14 \%$. The wet lands lost surface equal to $0.4 \%$. The surface, which was, converted into urban and industrial areas represent $2.6 \%$ and plantations $1.6 \%$. The main driver of land use change is the plantations because the Bio-Bio Region has the largest amount of surface area (858,592 ha) of the country with exotic plantations.
\end{abstract}

Keywords: monitoring; territory; plantations.

1 Estadístico, MSc., Profesor Asociado del Instituto de Estadística, Facultad de Ciencias Económicas y Administrativas, Universidad Austral de Chile, Los Laureles 35, Campus Isla Teja, CEP 5110027, Valdivia, Chile. gastonvergara@uach.cl

2 Ingeniero Forestal, Dr., Profesor Titular del Instituto de Bosque y Sociedad, Facultad de Ciencias Forestales y Recursos Naturales, Universidad Austral de Chile, Av. Dr. Eduardo Tallman s/n, Campus Isla Teja, CEP 5110027, Valdivia, Chile.vsandova@uach.cl

3 Ingeniero Forestal, Dr., Profesor Titular del Departamento de Ingeniería Forestal, Universidad de Córdoba, Campus de Rabanales Edificio Leonardo Da Vinci Carretera Nacional IV Km 396, CEP 14071, Córdoba, España. mc1hemam@uco.es

Recebido para publicação em 22/03/2015 e aceito em 24/01/2018

Ci. Fl., v. 28, n. 4, out. - dez., 2018 


\section{INTRODUCCIÓN}

El cambio del uso del suelo es una de las grandes preocupaciones de los científicos e investigadores durante las últimas décadas, este tipo de factor ejerce alteración en los ciclos y procesos naturales, repercutiendo finalmente en el cambio global y reducción de la diversidad biótica, alteración provocada por la interacción inadecuada entre el hombre y su medio ambiental (LAMBIN et al., 1999; LAMBIN; GEIST; LEPERS, 2003; TURNER; LAMBIN; REENBERG, 2007).

Uno de los grandes cambios de uso del suelo tiene relación con la deforestación, la cual se refleja en la intervención de los bosques por parte del ser humano, generando finalmente un ambiente antrópico, debido a la transformación de la cobertura del terreno y la degradación e intensificación del uso del suelo (ALVES 2002, LAMBIN; GEIST; LEPERS, 2003; ECHEVERRÍA et al., 2006).

La destrucción de los bosques tropicales y templados, sumado a ello el deterioro de la biodiversidad que albergan, trae como consecuencia la desaparición de gran parte de los recursos capaces de capturar carbono y controlar el calentamiento global (SHEERAN, 2006).

A nivel global, se estima que en las últimas décadas los cambios de uso del suelo han provocado grandes transformaciones a nuestros sistemas ambientales, estos impactos se centran principalmente en las transformaciones y muchas veces desaparición de los ecosistemas boscosos y praderas naturales, los cuales fueron transformados y habilitándolos para el desarrollo de la agricultura, ganadería, forestal y áreas urbanas e industriales (SALA et al., 2000; RUDEL et al., 2005).

Los estudios desarrollados en Chile señalan que existe un desacoplamiento entre la sociedad y los ecosistemas a nivel local, influyendo principalmente en los bosques templados, los cuales están siendo explotados en primera instancia para satisfacer la demanda internacional de productos asociados a la madera y papel, y en segundo lugar la habilitación de terrenos agrícolas y preparación de praderas (ECHEVERRÍA et al., 2006; 2008). Otros estudios recientes desarrollados en el Cetro Sur de Chile, que explican el cambio de la cobertura de uso de suelo, cuantificando la transición del bosque nativo, indican en primer lugar que la transición es hacia matorrales $(29 \%)$ y plantaciones forestales $(27 \%)$, principalmente Pinus radiata (ALTAMIRANO; LARA, 2010). La forma de expansión de las plantaciones forestales en su proceso inicial en Chile, es decir, hasta 1990 obedeció a patrones bien establecidos y que se relacionaban con los tipos de suelos con aptitud forestal y pendientes pronunciadas, patrones que posteriormente perdieron importancia, generando una expansión del uso del suelo para esta actividad forestal en forma anárquica y predominante en toda la región (NAHUELHUAL et al., 2012).

Estudiar los cambios en la cobertura del uso del suelo es esencial para determinar las tendencias que tendrán factores tales como: la deforestación, desertificación, degradación, cambios en la vegetación natural y la pérdida de biodiversidad en una determinada región (LAMBIN; GEIST; LEPERS, 2003).

Con el objetivo de cuantificar y describir el cambio de la cobertura de uso del suelo en dos periodos de tiempo, se construyen matrices de cambio que explican las transiciones de cambios entre los diferentes tipos de usos, previa interpretación de lo ocurrido a nivel de cobertura de suelo en cada periodo estudiado, utilizando para su identificación imágenes satelitales y fotografías aéreas (SANDOVAL; REAL, 2005; ALTAMIRANO; LARA, 2010).

En la presente investigación se evaluó en una escala espacial y temporal la dinámica de los cambios de uso del suelo ocurridos en la Región del Bio-Bio en el centro-sur de Chile, por ser la más poblada e industrializada y que presenta las tasas de forestación más alta del país (37\%) (INSTITUTO FORESTAL, 2007), cuantificando la dinámica del cambio de las coberturas de uso del suelo. Los objetivos principales de este trabajo corresponde específicamente a:

a) Construir indicadores que permitan describir los cambios de la cobertura de uso del suelo en la Región del Bio- Bio.

b) Realizar un análisis de los cambios de la cobertura de uso del suelo en la Región del Bio- Bio a nivel global y a nivel de provincia. 


\section{MATERIALES Y MÉTODOS}

\section{Área en estudio}

La Región del Bio-Bio se encuentra ubicada en el centro sur de Chile, limitada al Norte por la Región del Maule, al Sur por la Región de la Araucanía, al Este con la República Argentina y al Oeste con el Océano Pacífico (Figura 1), se localiza entre $\operatorname{los} 36^{\circ}$ y los $38^{\circ} 30^{\prime}$ de latitud sur, y desde los $71^{\circ}$ hasta los $73^{\circ} 40^{\prime}$ de longitud oeste, a una altitud promedio de $1.430 \mathrm{msnm}$. Está estructurada administrativamente por cuatro provincias y el territorio insular lo componen las islas Quiriquina, Santa María y La Mocha. Posee una población proyectada al 2016 de 2.127 .902 habitantes (INSTITUTO NACIONAL DE ESTADÍSTICAS, 2002), con una tasa de crecimiento intercensal de $0,7 \%$. La superficie es de 3.706 .003 ha, distribuidas en las provincias de Concepción con 346.300 ha, Bio-Bio 1.505.432 ha, Arauco 544.470 ha y Ñuble con 1.309.800 ha respectivamente (CORPORACIÓN NACIONAL FORESTAL, 1999).

El clima corresponde a una transición entre el clima mediterráneo y el templado húmedo con lluvias intermitentes. Las temperaturas medias oscilan entre $\operatorname{los} 12,4^{\circ} \mathrm{C}$ y $\operatorname{los} 14^{\circ} \mathrm{C}$ en la costa y las zonas cordilleranas. Las precipitaciones son superiores a los $1.000 \mathrm{~mm}$ anuales, concentrándose principalmente entre los meses de abril a noviembre.

La geomorfología de la región no escapa a la que se presenta en todo el país, esto es, cordillera-depresión intermedia-planicies, entre la variedad de los ambientes naturales, destaca el sistema insular, que enriquecen tanto la vegetación como sus paisajes.

La Región del Bio-Bio representa la mayor cantidad en superficie de bosques plantados del país, con 858.592 ha (principalmente Eucalyptus 240.473 ha y P. radiata 610.124 ha), representando el 37,3\% del total del país, seguida solo por la Regiones del Maule con un 18,8\% (principalmente Eucalyptus 38.196 ha y P. radiata 389.434 ha) y La Araucanía con un 19,2 \% (principalmente Eucalyptus 168.019 ha y P. radiata $262.430 \mathrm{ha}$ ) (INSTITUTO FORESTAL, 2008).

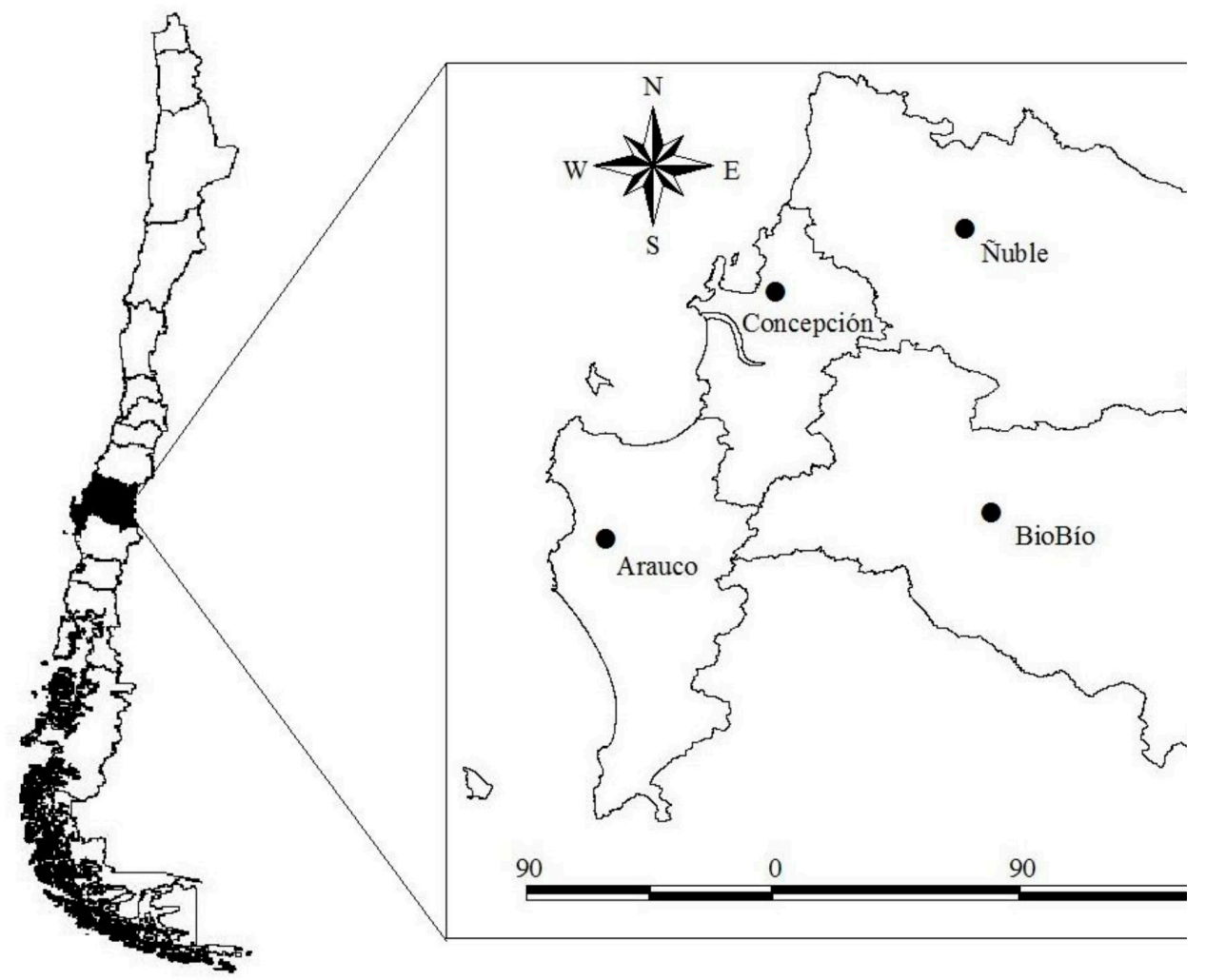

FIGURA 1: Mapa del área de estudio, región y provincias.

FIGURE 1: Map of the study area, region and provinces. 


\section{Material empleado}

Se utilizó para el primer periodo a nivel de región la información base proveniente del proyecto denominado "Catastro de los Recursos Vegetacionales Nativos de Chile 1998", en el cual se usaron cartas temáticas para generar una representación cartográfica detallada del uso de la tierra, en relación a vegetación y bosques a lo largo de todo el territorio nacional continental. Se interpretaron aproximadamente 50.000 fotografías aéreas. Se describieron en terreno alrededor del $50 \%$ de los polígonos con bosque nativo y mediante un sketchmaster se traspasaron los datos desde las fotos aéreas a las cartas topográficas base, en escala 1:50.000, 1:100.000 y 1:250.000, según disponibilidad de cada región. Posteriormente y a medida que se dispuso de nuevas y mejores tecnologías, se realizaron correcciones respecto a la identificación de polígonos, exactitud de los límites y la escala de representación (CORPORACIÓN NACIONAL FORESTAL, 1999). Para el segundo periodo, año 2008, se usaron fotografías aéreas, obtenidas del Servicio Aéreo Fotogramétrico (SAF) a una escala 1:10.000. Las fotografías aéreas se escanearon y georreferenciaron al sistema de coordenadas UTM WGS-84 (18S), utilizando puntos de control obtenidos de las coberturas vectoriales provenientes del catastro y monitoreo nacional de uso de suelo (CORPORACIÓN NACIONAL FORESTAL, 1999). Usando las imágenes escaneadas y georreferenciadas del año 2008, se digitalizaron los polígonos de cambio, utilizando el software ArcGis 10, asignándole a cada polígono de cambio un número de identificación único, además el número de polígono del periodo anterior.

La tipología para la clasificación de la cobertura de uso del suelo se basó en la metodología desarrollada por el Centro de Estudios Fitosociológicos y Ecológicos L. Emberger (CEPE de Montpellier), conocida como carta de ocupación de la tierra (SANDOVAL, 2008). Las principales clasificaciones de uso del suelo fueron: (1) Áreas Urbanas e Industriales, (2) Terrenos Agrícolas, (3) Praderas y Matorrales, (4.1) Plantaciones, (4.2) Bosque Nativo, (4.3) Bosque Mixto, (5) Humedales, (6) Áreas Desprovistas de Vegetación, (7) Nieves y Glaciales, (8) Cuerpos de Agua, (9) Áreas no reconocidas.

\section{Metodología}

Para la recolección de la información de terreno con la interpretación de los polígonos identificados cartográficamente y cambios de uso del suelo en el segundo periodo, se utilizaron formularios que permitieron describir el terreno en relación al uso actual, causales de cambio, árboles, arbustos y especies (SANDOVAL, 2008). Los formularios se transfirieron a una base datos, a través de un Sistema de Información construido para el monitoreo y actualización del cambio de uso del suelo (VERGARA; SANDOVAL, 2010). La información de terreno se validó y posteriormente se unió a la base de datos gráfica a través del identificador único poligonal, posteriormente utilizando ARCGIS 10 (ARCGIS, 2001), se cruzaron ambas coberturas e incluyendo además una cobertura con los límites administrativos territoriales, el producto que se obtiene numéricamente corresponde a una base de datos, la cual se incorpora al Sistema de Información (VERGARA; SANDOVAL, 2010) obteniéndose las diferentes matrices que explican la evolución del cambio y la transición de coberturas (DEZZEO; SENDRA, 2008).

La matriz de cambio permitió cuantificar la evolución de uso del suelo, ésta consiste en una matriz simétrica, en filas y columnas se representan los diferentes tipos de coberturas de clasificación del suelo y al interior en cada celda está presente la superficie de los usos que experimentaron cambios en el periodo 1998 y 2008 (AGUAYO et al., 2009). Con operaciones de sumas y restas de las filas obtenemos las pérdidas y al realizar las mismas operaciones con las columnas se obtienen las ganancias del periodo estudiado.

Para calcular la tasa de cambio se utilizó la fórmula de interés compuesto (PUYRAVAUD, 2003).

$$
P=\frac{100}{t_{2}-t_{1}} \ln \left(\frac{A_{2}}{A_{1}}\right)
$$

Dónde: $\mathrm{P}=$ tasa de cambio por año en porcentaje; $\mathrm{A}_{1}=$ superficie en hectáreas de la clase de uso en el tiempo inicial; $\mathrm{A}_{2}$ $=$ superficie en hectáreas de la clase de uso en el tiempo final; $\mathrm{t}_{1}=\mathrm{año}$ de evaluación inicial; $\mathrm{t}_{2}=$ año de evaluación final. 


\section{RESULTADOS}

Al analizar cada periodo podemos observar que el año 1998 los terrenos agrícolas y plantaciones tienen el predominio del uso en la región (Tabla 1, Figura 2), cada uno con un $26 \%$, representando entre ambos más del $50 \%$ de la superficie regional, en un segundo lugar se encuentra el bosque nativo (21\%) y en tercer lugar las praderas y matorrales $(17,2 \%)$.

TABLA 1: Superficie, porcentaje y tasa de cambio de coberturas de uso de suelo para los años 1998 y 2008 en la Región del Bio-Bio.

TABLE 1: Area, percentage and rate of change of land use coverage for the years 1998 and 2008 in the Bio-Bio Region.

\begin{tabular}{|c|c|c|c|c|c|c|c|}
\hline \multirow{2}{*}{ Tipos del Uso } & \multicolumn{2}{|c|}{1998} & \multicolumn{2}{|c|}{2008} & \multicolumn{2}{|c|}{ Cambio } & \multirow{2}{*}{$\begin{array}{c}\text { Tasa de Cambio } \\
\text { Anual } \\
\end{array}$} \\
\hline & ha & $\%$ & ha & $\%$ & ha & $\%$ & \\
\hline $\begin{array}{l}1 \text { Áreas urbanas e } \\
\text { Industriales }\end{array}$ & 25,803 & 0,7 & 35,686 & 1,0 & 9,882 & 38,3 & 3,2 \\
\hline 2 Terrenos agrícolas & 965,145 & 26,0 & 829,508 & 22,4 & $-135,637$ & $-14,1$ & $-1,5$ \\
\hline 3 Praderas y matorrales & 637,163 & 17,2 & 498,496 & 13,5 & $-138,667$ & $-21,8$ & $-2,5$ \\
\hline 4.1 Plantaciones & 958,697 & 25,9 & 1.227 .788 & 33,1 & 269,091 & 28,1 & 2,5 \\
\hline 4.2 Bosque nativo & 776,436 & 21,0 & 768,552 & 20,7 & $-7,883$ & $-1,0$ & $-0,1$ \\
\hline 4.3 Bosque mixto & 53,294 & 1,4 & 56,642 & 1,5 & 3,348 & 6,3 & 0,6 \\
\hline 5 Humedales & 12,027 & 0,3 & 11,595 & 0,3 & -432 & $-3,6$ & $-0,4$ \\
\hline $\begin{array}{c}6 \text { Áreas desprovistas de } \\
\text { vegetación }\end{array}$ & 133,976 & 3,6 & 132,518 & 3,6 & $-1,458$ & $-1,1$ & $-0,1$ \\
\hline 7 Nieves y glaciares & 90,692 & 2,4 & 90,468 & 2,4 & -224 & $-0,2$ & 0,0 \\
\hline 8 Cuerpos de agua & 52,558 & 1,4 & 54,742 & 1,5 & 2,184 & 4,2 & 0,4 \\
\hline 9 Áreas no reconocidas & 211 & 0,0 & 8 & 0,0 & -203 & $-96,1$ & $-32,6$ \\
\hline Total & 3.706 .003 & & 3.706 .003 & & & & \\
\hline
\end{tabular}

Para el segundo periodo (año 2008), los terrenos agrícolas bajan en superficie, llegando a un 22,4 \% de representatividad del territorio regional (Tabla 1, Figura 2), con una tasa negativa en el periodo de cambio del 1,5\% anual, las praderas y matorrales presentan también una disminución en su superficie, llegando a una representación del $13,5 \%$, con tasa de cambio negativa de 2,5\% anual, sin embargo la superficie de plantaciones forestales presentan un incremento en este nuevo periodo, llegando a cubrir el 33,1\% de la superficie del territorio de la región, lo cual representa una tasa anual de incremento del $2,5 \%$, por otro lado las áreas urbanas e industriales, a pesar de su baja representación sobre el territorio, también tienen un incremento pasando de $0,7 \%$ al $1,0 \%$ del territorio, incrementándose en el periodo estudiado un 38,3\%, con una tasa de crecimiento anual del $3,2 \%$. 


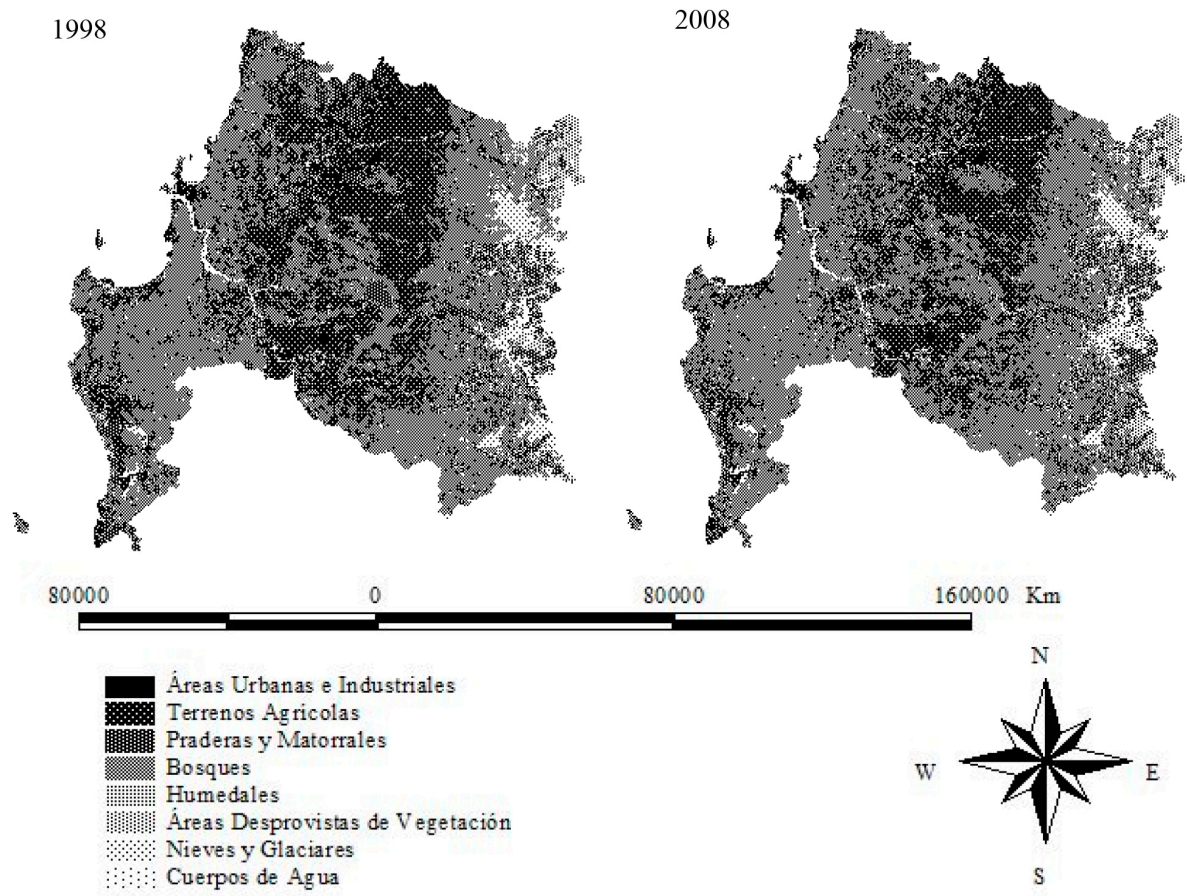

FIGURA 2: Coberturas de uso del suelo obtenidas del monitoreo 1998 y 2008.

FIGURE 2: Land use covers obtained from monitoring 1998 and 2008.

TABLA 2: Matriz de cambio de cobertura de uso del suelo entre los años 1998 y 2008, en la Región del Bio-Bio. TABLE 2: Matrix cover change in land use between 1998 and 2008, in the Bio-Bio Region.

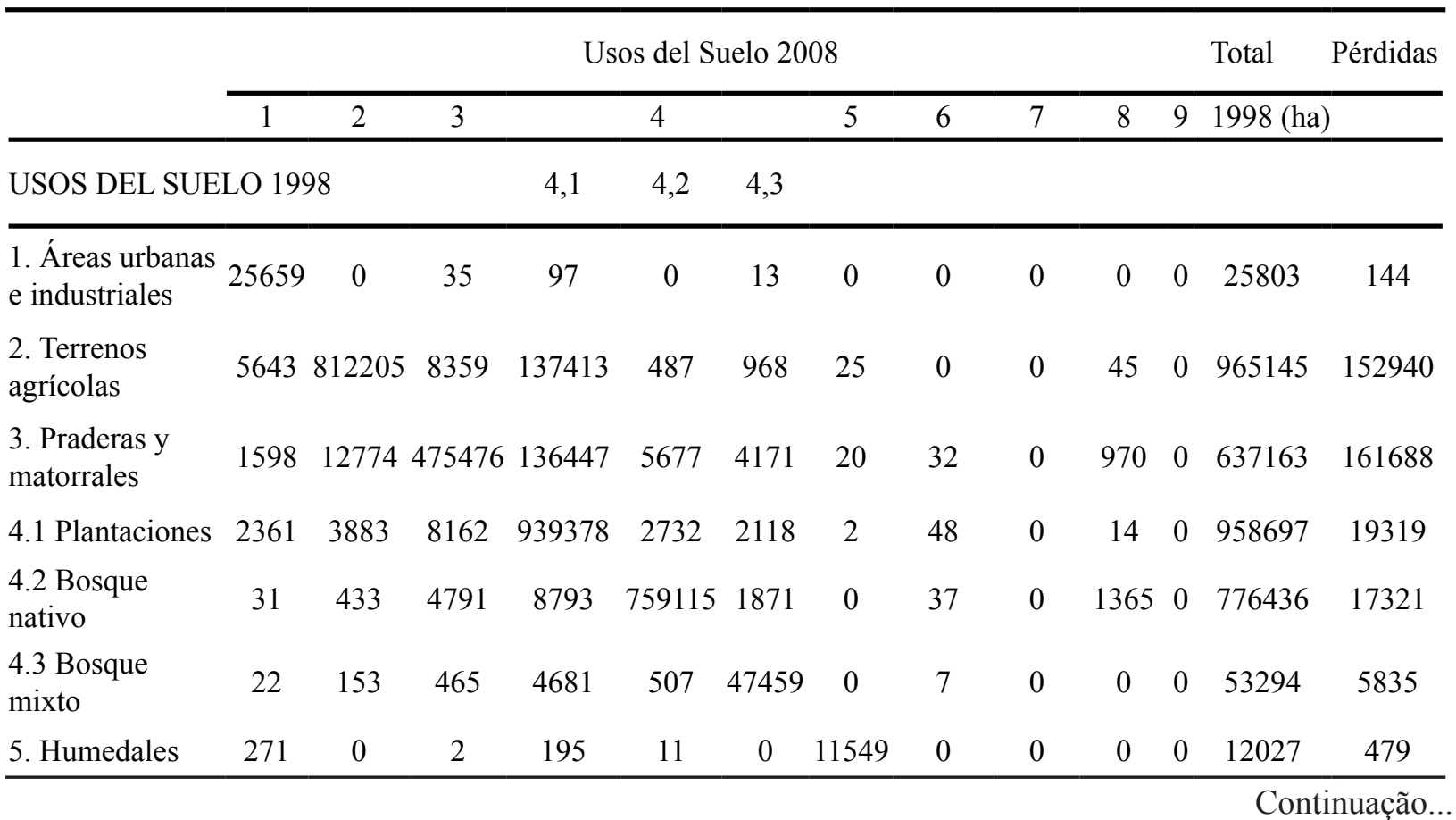


TABELA 2: Continua...

TABLE 2 Continued...

\begin{tabular}{|c|c|c|c|c|c|c|c|c|c|c|c|c|c|}
\hline & \multicolumn{11}{|c|}{ Usos del Suelo 2008} & Total & \multirow[t]{2}{*}{ Pérdidas } \\
\hline & 1 & 2 & 3 & & 4 & & 5 & 6 & 7 & 8 & 9 & 1998 (ha) & \\
\hline \multicolumn{4}{|c|}{ USOS DEL SUELO 1998} & 4,1 & 4,2 & 4,3 & & & & & & & \\
\hline $\begin{array}{l}\text { 6. Áreas } \\
\text { desprovistas de } \\
\text { vegetación }\end{array}$ & 84 & 0 & 919 & 557 & 5 & 25 & 0 & 132386 & 0 & 0 & 0 & 133976 & 1590 \\
\hline $\begin{array}{l}\text { 7. Nieves y } \\
\text { glaciares }\end{array}$ & 0 & 0 & 216 & 0 & 0 & 0 & 0 & 8 & 90468 & 0 & 0 & 90692 & 224 \\
\hline $\begin{array}{l}\text { 8. Cuerpos de } \\
\text { agua }\end{array}$ & 16 & 47 & 71 & 57 & 0 & 18 & 0 & 0 & 0 & 52349 & 0 & 52558 & 210 \\
\hline $\begin{array}{l}9 \text { Áreas no } \\
\text { reconocidas }\end{array}$ & 0 & 13 & 0 & 171 & 19 & 0 & 0 & 0 & 0 & 0 & 8 & 211 & 203 \\
\hline Total 2008 (ha) & 356868 & 829494 & 498496 & 1227616 & 768534 & 56642 & 1159 & 132518 & 90468 & 54742 & & 3706003 & \\
\hline Ganancias & 10027 & 17290 & 23021 & 288238 & 9419 & 9183 & 46 & 132 & 0 & 2394 & & & \\
\hline
\end{tabular}

En relación a los terrenos agrícolas un 84,2 \% se mantuvo el año 2008 (Tabla 2, Figura 3). La superficie que se perdió en el periodo estudiado, se reemplazó principalmente por plantaciones forestales en un $14 \%$ y otro porcentaje $(0,6 \%)$ se transfirió a las áreas urbanas e industriales.

Parte de la superficie de terrenos agrícolas que se encuentra presente el año 2008, corresponde a superficie que el año 1998 pertenecían a la clase de uso áreas no reconocidas 6,2 \% y en un porcentaje menor 2,0 \% provienen de praderas y matorrales (Tabla 2, Figura 3 ).

La superficie perteneciente al tipo de uso plantaciones forestales presentes el año 2008 (Tabla 2, Figura 3), provienen en un $21,0 \%$ de superficie que el año 1998 pertenecían a praderas y matorrales, también las plantaciones ocuparon superficie de terrenos agrícolas (14\%), áreas no reconocidas ( $81 \%$ ), bosque nativo (11\%), bosque mixto $(8,8 \%)$ e incluso el tipo de uso humedales aporta el $1,4 \%$.

El 98,7 \% de la superficie de bosque nativo que existía el año 1998, se mantuvo el año 2008, la superficie que se perdió, se reemplazó principalmente por plantaciones, esto es, un $1,1 \%$ y un $0,6 \%$ se reemplazó por praderas y matorrales (Tabla 2, Figura 3).

De la superficie de bosque nativo existente el año 2008, un $8,9 \%$ corresponde a terrenos que el año 1998 pertenecía a la clase de uso áreas no reconocidas, en igual situación la clase de uso praderas y matorrales aporta con un $0,9 \%$. 


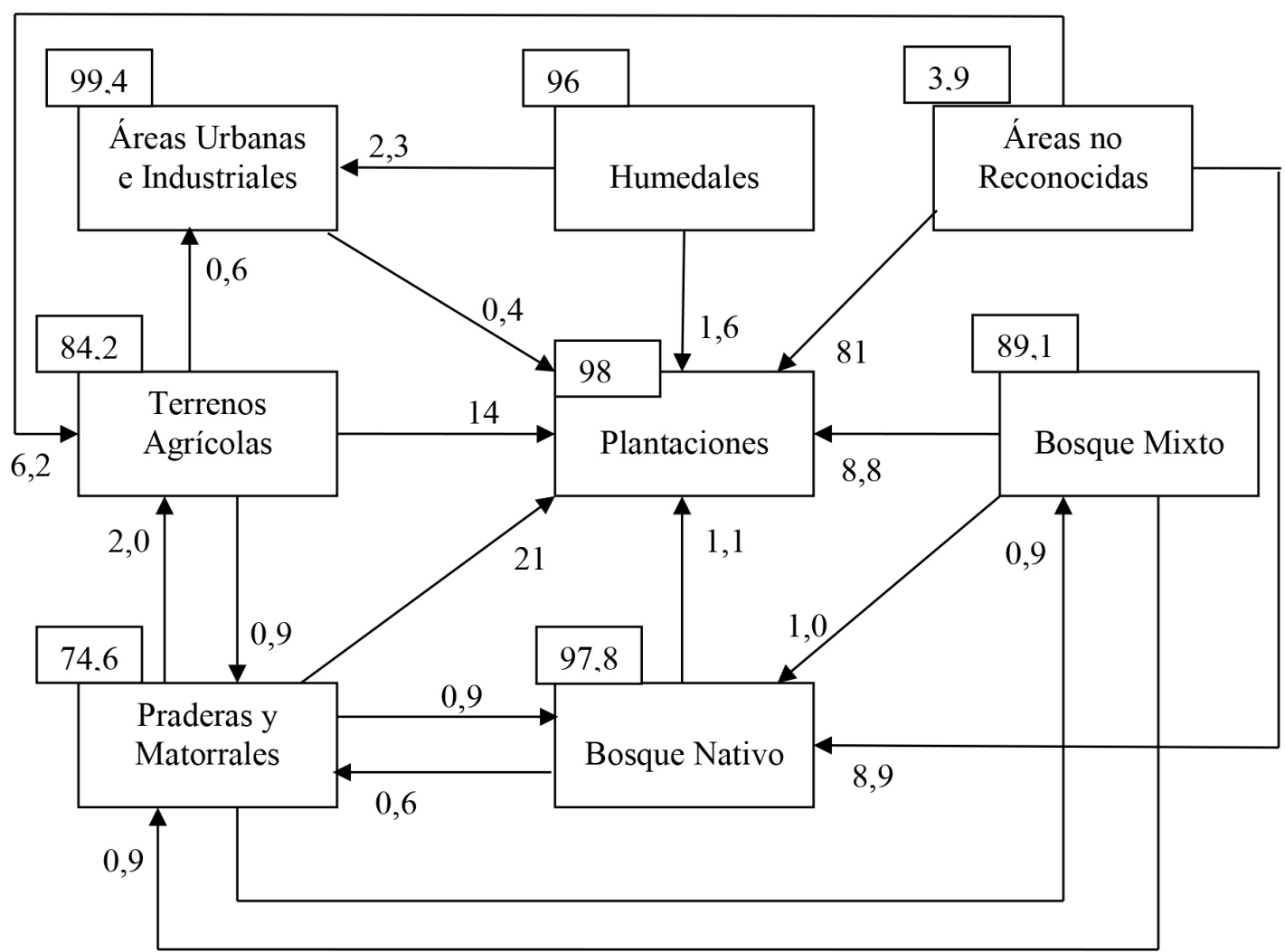

FIGURA 3: Dirección del cambio entre las diferentes coberturas de usos del suelo entre los años 1998 y 2008. Por una baja movilidad porcentual, no incluye las categorías: (6) áreas desprovistas de vegetación, (7) nieves y glaciales y (8) cuerpos de agua. Los valores en rectángulos pequeños corresponden a porcentaje de superficie que no experimentó cambios durante el periodo de estudio; por ejemplo un 74,6\% de la superficie de praderas y matorrales se mantuvieron como tal en el periodo 1998 y 2008.

FIGURE 3: Change direction between the different land use covers from 1998 to 2008. For a low percentage, mobility excludes categories: (6) areas without vegetation (7) snow and glaciers (8) bodies of water. The values in small rectangles correspond to the percentage of surface that presented changes during the study period (e.g. $74.6 \%$ of the area of plains and scrublands were maintained like in the period 1998-2008). 
TABLA 3: Área (en hectáreas), porcentaje y tasa de cambio de coberturas de uso de suelo entre los años 1998 y 2008, en la provincia de Arauco, Región del Bio-Bio.

TABLE 3: Area (in hectares), percentage and rate change of land use covers between 1998 and 2008, in Arauco province, Bio-Bio Region.

\begin{tabular}{|c|c|c|c|c|c|c|c|}
\hline \multirow[b]{2}{*}{ Tipos del Uso } & \multicolumn{2}{|c|}{1998} & \multicolumn{2}{|c|}{2008} & \multicolumn{2}{|c|}{ Cambio } & \multirow{2}{*}{$\begin{array}{c}\text { Tasa } \\
\text { Cambio } \\
\text { Anual }\end{array}$} \\
\hline & ha & $\%$ & ha & $\%$ & ha & $\%$ & \\
\hline $\begin{array}{l}1 \text { Áreas } \\
\text { urbanas e } \\
\text { industriales }\end{array}$ & $2.656,6$ & 0,5 & $3.159,4$ & 0,6 & 502,9 & 18,9 & 1,7 \\
\hline $\begin{array}{l}2 \text { Terrenos } \\
\text { agrícolas }\end{array}$ & $64.601,4$ & 11,9 & $57.882,1$ & 10,6 & $-6.719,2$ & $-10,4$ & $-1,1$ \\
\hline $\begin{array}{l}3 \text { Praderas y } \\
\text { matorrales }\end{array}$ & $100.643,2$ & 18,5 & $68.749,6$ & 12,6 & $-31.893,6$ & $-31,7$ & $-3,8$ \\
\hline $\begin{array}{l}4.1 \\
\text { Plantaciones }\end{array}$ & $247.938,1$ & 45,5 & $284.761,7$ & 52,3 & $36.823,6$ & 14,9 & 1,4 \\
\hline $\begin{array}{l}4.2 \text { Bosque } \\
\text { nativo }\end{array}$ & $94.781,0$ & 17,4 & $95.081,7$ & 17,5 & 300,7 & 0,3 & 0,0 \\
\hline $\begin{array}{l}4.3 \text { Bosque } \\
\text { mixto }\end{array}$ & $11.772,8$ & 2,2 & $14.161,5$ & 2,6 & $2.388,7$ & 20,3 & 1,8 \\
\hline 5 Humedales & $5.232,3$ & 1,0 & $5.162,5$ & 0,9 & $-69,8$ & $-1,3$ & $-0,1$ \\
\hline $\begin{array}{l}6 \text { Áreas } \\
\text { desprovistas } \\
\text { de vegetación }\end{array}$ & $7.563,3$ & 1,4 & $6.239,5$ & 1,1 & $-1.323,8$ & $-17,5$ & $-1,9$ \\
\hline $\begin{array}{l}7 \text { Nieves y } \\
\text { glaciares }\end{array}$ & 0,0 & 0,0 & 0,0 & 0,0 & 0,0 & 0,0 & 0,0 \\
\hline $\begin{array}{l}8 \text { Cuerpos de } \\
\text { agua }\end{array}$ & $9.281,9$ & 1,7 & $9.272,4$ & 1,7 & $-9,4$ & $-0,1$ & 0,0 \\
\hline $\begin{array}{l}9 \text { Áreas no } \\
\text { reconocidas }\end{array}$ & 0,0 & 0,0 & 0,0 & 0,0 & 0,0 & 0,0 & 0,0 \\
\hline Total & $544.470,5$ & & $544.470,5$ & & & & \\
\hline
\end{tabular}

Al analizar ambos periodos en la provincia de Arauco, podemos observar que el año 1998 las plantaciones forestales tienen el predominio del uso de la superficie (Tabla 3), ocupando el $46 \%$ del territorio de la provincia, en un segundo lugar se encuentra las praderas y matorrales con un 18,5\%, seguido en porcentaje similar por el bosque nativo (17,4 \%). El año 2008 continúa predominado la cobertura de uso plantaciones, aumentando a 52,3\% con una tasa de crecimiento anual del $1,4 \%$, la superficie de bosque mixto presenta un pequeño incremento llegando a $2,6 \%$ con una tasa de crecimiento anual del 1,8 \%, la cobertura de uso asociada a praderas y matorrales disminuyó $(12,6 \%)$ con una tasa decreciente anual de 3,8 $\%$, el bosque nativo mantuvo su superficie en ambos periodos, las áreas urbanas en los dos periodos tienen baja representación respecto a la superficie total, sin embargo el año 2008 se produce un incremento con una tasa anual del 1,7\%, los terrenos agrícolas disminuyeron su superficie con una representación territorial del $10,6 \%$ y una tasa negativa anual del $1,1 \%$. 
TABLA 4: Área (en hectáreas), porcentaje y tasa de cambio de coberturas de uso de suelo entre los años 1998 y 2008 en la provincia de Bio-Bio, Región del Bio-Bio.

TABLE 4: Area (in hectares), percentage and rate change of land use covers between 1998 and 2008, in Bio-Bio province, Bio-Bio region.

\begin{tabular}{|c|c|c|c|c|c|c|c|}
\hline \multirow{2}{*}{ Tipos del Uso } & \multicolumn{2}{|c|}{1998} & \multicolumn{2}{|c|}{2008} & \multicolumn{2}{|c|}{ Cambio } & \multirow{2}{*}{$\frac{\text { Tasa }}{\text { Cambio Anual }}$} \\
\hline & ha & $\%$ & ha & $\%$ & ha & $\%$ & \\
\hline $\begin{array}{l}1 \text { Áreas urbanas e } \\
\text { industriales }\end{array}$ & 4,763 & 0,3 & 7,9 & 0,5 & 3,137 & 65,9 & 5,1 \\
\hline 2 Terrenos agrícolas & 350,264 & 23,3 & 292,354 & 19,4 & $-57,911$ & $-16,5$ & $-1,8$ \\
\hline $\begin{array}{l}3 \text { Praderas y } \\
\text { matorrales }\end{array}$ & 275,839 & 18,3 & 241,341 & 16,0 & $-34,498$ & $-12,5$ & $-1,3$ \\
\hline 4.1 Plantaciones & 302,862 & 20,1 & 393,933 & 26,2 & 91,071 & 30,1 & 2,6 \\
\hline 4.2 Bosque nativo & 431,556 & 28,7 & 426,227 & 28,3 & $-5,329$ & $-1,2$ & $-0,1$ \\
\hline 4.3 Bosque mixto & 14,277 & 0,9 & 16,061 & 1,1 & 1,784 & 12,5 & 1,2 \\
\hline 5 Humedales & 1,389 & 0,1 & 1,389 & 0,1 & 0 & 0,0 & 0,0 \\
\hline $\begin{array}{l}6 \text { Áreas desprovistas } \\
\text { de vegetación }\end{array}$ & 58,684 & 3,9 & 58,651 & 3,9 & -33 & $-0,1$ & 0,0 \\
\hline 7 Nieves y glaciares & 42,856 & 2,8 & 42,632 & 2,8 & -224 & $-0,5$ & $-0,1$ \\
\hline 8 Cuerpos de agua & 22,765 & 1,5 & 24,938 & 1,7 & 2,172 & 9,5 & 0,9 \\
\hline $\begin{array}{l}9 \text { Áreas no } \\
\text { reconocidas }\end{array}$ & 177 & 0,0 & 8 & 0,0 & -169 & $-95,4$ & $-30,8$ \\
\hline Total & 1.505 .432 & & 1.505 .432 & & & & \\
\hline
\end{tabular}

En la provincia del Bio-Bio el año 1998 (Tabla 4) la principal cobertura de uso corresponde a bosque nativo con un $28,3 \%$, en segundo lugar se encuentran los terrenos agrícolas con un $23 \%$ y posteriormente los usos asociados las plantaciones forestales (20,1\%), y praderas y matorrales con un 18,3\% respectivamente. Durante el año 2008 solamente presentaron un incremento positivo los usos relacionados con áreas urbanas e industriales con una tasa anual de 5,1\% y las plantaciones con una tasa de crecimiento del 2,6 \% al año, el resto de los usos presentaron tasas de incremento negativas.

TABLA 5: Área (en hectáreas), porcentaje y tasa de cambio de coberturas de uso de suelo para los años 1998 y 2008 en la provincia de Concepción, Región del Bio-Bio.

TABLE 5: Area (in hectares), percentage and rate change of land use covers between 1998 and 2008, in Concepción province, Bio-Bio Region.

\begin{tabular}{llcccccc}
\hline \multirow{2}{*}{ Tipos del Uso } & \multicolumn{2}{c}{1998} & \multicolumn{2}{c}{2008} & \multicolumn{2}{c}{ Cambio } & Tasa \\
\cline { 2 - 8 } & ha & $\%$ & ha & $\%$ & ha & $\%$ & Cambio Anual \\
\hline 1 Áreas urbanas e industriales 12,746 & 3,7 & 16,213 & 4,7 & 3,468 & 27,2 & 2,4 \\
2 Terrenos agrícolas & 52,961 & 15,3 & 38,497 & 11,1 & $-14,465$ & $-27,3$ & $-3,2$ \\
3 Praderas y matorrales & 47,406 & 13,7 & 34,669 & 10,0 & $-12,737$ & $-26,9$ & $-3,1$ \\
4.1 Plantaciones & 174,445 & 50,4 & 198,959 & 57,5 & 24,514 & 14,1 & 1,3 \\
4.2 Bosque nativo & 26,856 & 7,8 & 26,678 & 7,7 & -177 & $-0,7$ & $-0,1$ \\
4.3 Bosque mixto & 14,967 & 4,3 & 14,826 & 4,3 & -142 & $-0,9$ & $-0,1$ \\
5 Humedales & 4,305 & 1,2 & 3,959 & 1,1 & -346 & $-8,0$ & $-0,8$ \\
6 Áreas desprovistas de & 1,172 & 0,3 & 1,121 & 0,3 & -51 & $-4,3$ & $-0,4$ \\
vegetación & & & & & & & Contingca.,.
\end{tabular}


TABELA 5: Continua...

TABLE 5 Continued...

\begin{tabular}{lllccccc}
\hline \multirow{2}{*}{ Tipos del Uso } & \multicolumn{2}{c}{1998} & \multicolumn{2}{c}{2008} & \multicolumn{2}{c}{ Cambio } & Tasa \\
\cline { 2 - 8 } & ha & $\%$ & ha & $\%$ & ha & $\%$ & Cambio Anual \\
\hline 7 Nieves y glaciares & 0 & 0,0 & 0 & 0,0 & 0 & 0,0 & 0,0 \\
8 Cuerpos de agua & 11,409 & 3,3 & 11,379 & 3,3 & -30 & $-0,3$ & 0,0 \\
9 Áreas no reconocidas & 34 & 0,0 & 0 & 0,0 & -34 & $-100,0$ & 0,0 \\
\hline Total & 346,3 & 346,3 & \\
\hline
\end{tabular}

En la provincia de Concepción se presenta la misma tendencia que el resto de las provincias, es decir, las únicas coberturas de uso del suelo que crecen, corresponden a las áreas urbanas e industriales y plantaciones forestales, en este caso con tasas anuales de crecimiento del 2,4 \% y 1,3\% respectivamente. Para el caso de los usos terrenos agrícolas, y praderas y matorrales las tasas anuales de disminución territorial son bastante significativas, alrededor del 3,2 \% (Tabla 5).

TABLA 6: Área (en hectáreas), porcentaje y tasa de cambio de coberturas de uso de suelo para los años 1998 y 2008 en la provincia de Ñuble, Región del Bio-Bio.

TABLE 6: Area (in hectares), percentage and rate change of land use covers between 1998 and 2008, in Nuble province, Bio-Bio Region.

\begin{tabular}{lccccccc}
\hline \multirow{2}{*}{ Tipos del Uso } & \multicolumn{2}{c}{1998} & \multicolumn{2}{c}{2008} & \multicolumn{2}{c}{ Cambio } & Tasa \\
\cline { 2 - 8 } & ha & $\%$ & ha & $\%$ & ha & $\%$ & $\begin{array}{c}\text { Cambio } \\
\text { Anual }\end{array}$ \\
\hline 1 Áreas urbanas e industriales & 5.638 & 0,4 & 8.413 & 0,6 & 2.775 & 49,2 & 4,0 \\
2 Terrenos agrícolas & 497.318 & 38,0 & 440.775 & 33,7 & -56.543 & $-11,4$ & $-1,2$ \\
3 Praderas y matorrales & 213.275 & 16,3 & 153.737 & 11,7 & -59.538 & $-27,9$ & $-3,3$ \\
4.1 Plantaciones & 233.452 & 17,8 & 350.134 & 26,7 & 116.682 & 50,0 & 4,1 \\
4.2 Bosque nativo & 223.244 & 17,0 & 220.565 & 16,8 & -2.678 & $-1,2$ & $-0,1$ \\
4.3 Bosque mixto & 12.277 & 0,9 & 11.594 & 0,9 & -683 & $-5,6$ & $-0,6$ \\
5 Humedales & 1.102 & 0,1 & 1.085 & 0,1 & -16 & $-1,5$ & $-0,2$ \\
6 Áreas desprovistas de vegetación & 66.557 & 5,1 & 66.507 & 5,1 & -50 & $-0,1$ & 0,0 \\
7 Nieves y glaciares & 47.836 & 3,7 & 47.836 & 3,7 & 0 & 0,0 & 0,0 \\
8 Cuerpos de agua & 9.103 & 0,7 & 9.154 & 0,7 & 51 & 0,6 & 0,1 \\
9 Áreas no reconocidas & 0 & 0,0 & 0 & 0,0 & 0 & 0,0 & 0,0 \\
\hline Total & 1.309 .800 & \multicolumn{10}{c}{1.309 .800} & \multicolumn{3}{c}{} \\
\hline
\end{tabular}

La provincia de Ñuble ha experimentado un gran crecimiento en la cobertura de plantaciones forestales, pasando en la ocupación territorial de un 17,8 \% el año 1998 a 26,7 \% el año 2008, lo cual representa una tasa de incremento anual del 4,1\%, una tendencia similar presentó la cobertura de uso áreas urbanas e industriales, pasando de $0,4 \%$ el año 1998 a $0,6 \%$ el 2008 con tasa de incremento anual del 4,0 \%. Las tasas más relevantes con incremento negativo y por lo tanto con pérdida de cobertura de uso, la representan praderas y matorrales con tasas de disminución anual de 3,3\% y los terrenos agrícolas con 1,3\% (Tabla 6).

En general al analizar las diferentes provincias se puede observar que tienen conductas similares en su predominio de las coberturas de usos, situación que no contrasta con lo observado a nivel regional, las coberturas de uso áreas urbanas e industriales y plantaciones forestales presentan tasas anuales de cambio en permanente incremento, por supuesto en desmedro de aquellas coberturas de uso fáciles de degradar 
como son los terrenos agrícolas, praderas y matorrales, bosque nativo y bosque mixto.

\section{DISCUSIÓN}

La tasa de crecimiento calculada de las áreas urbanas e industriales $(3,2 \%$ anual $)$, difiere notablemente de la obtenida por CORPORACIÓN NACIONAL FORESTAL (1999) de 1,5\%, en un periodo corto para los años 1994 y 1998, esto se debe principalmente a que la dinámica del territorio es distinta, sin embargo ésta es bastante similar (3,8\%) a la obtenida por AGUAYO et al. (2009) en su estudio realizado en las Regiones de Bio-Bio y La Araucanía, como así mismo las obtenidas también por AZÓCAR, SANHUEZA y HENRÍQUEZ (2003), AZOCAR et al. (2007) y HENRÍQUEZ, AZÓCAR y ROMERO (2006) para las ciudades de Chillan $(3,1 \%)$ y Los Ángeles $(3,4 \%)$.

La tasa de pérdida del bosque nativo $(0,1 \%$ anual $)$ se aproxima bastante a las estimaciones obtenidas por CORPORACIÓN NACIONAL FORESTAL (1999) en la Región del Bio-Bio, en un periodo corto para los años 1994 y 1998 (0,3 \%) y difiere de la obtenida por AGUAYO et al. (2009) de 1,6 \%, diferencia que se justifica por la extensión del territorio que se consideró para ambos estudios. Por otro lado existen antecedentes relevantes que indican que la pérdida de bosque nativo a causa de la sustitución por plantaciones exóticas debe ir disminuyendo (INSTITUTO FORESTAL, 2007). La tasa del incremento de las plantaciones de especies exóticas, especialmente plantaciones de Pinus radiata, fueron de 2,5\%, con una tasa de cambio de $28,1 \%$ cifras muy similares a las obtenidas por NAHUELHUAL et al. (2012) de un 22,8 $\%$ en periodos similares, la diferencia se justifica por un desfase en los periodos monitoreados y además el presente estudio incluye toda la región, establece un refinamiento más preciso de los tipos de usos del territorio monitoreados y su ratificación de visitas en terreno con brigadas especializadas.

Según los antecedentes obtenidos se le atribuye a las empresas forestales la principal causa de la deforestación, situación que ha disminuido últimamente debido a que los remanentes de bosque nativo se encuentran en sectores de difícil acceso con fuertes pendientes, altitudes por sobre los $800 \mathrm{~m}$, o al interior de áreas protegidas (ALTAMIRANO; LARA, 2010). Otros antecedentes que permiten avanzar en este sentido tienen relación con las conductas empresariales, debido a que desde el año 1997 comenzó un proceso de certificación de las empresas forestales, comprometiéndose éstas al manejo sustentable de los recursos forestales del país (LARA; ECHEVERRÍA; REYES, 2002). Otro factor importante tiene relación con la promulgación de la nueva Ley de fomento y recuperación del bosque nativo, la cual incorpora incentivos económicos para los propietarios de bosques nativos (LARA et al., 2003).

Los terrenos agrícolas presentan una tasa de pérdida anual del 1,5\%, las principales causa se relacionan con el alto subsidio estatal que incentiva a los propietarios a la habilitación de estos tipos de usos para la actividad forestal y en un porcentaje menor hacia la expansión de las ciudades. Estudios previos en la Región del Bio-Bio (CORPORACIÓN NACIONAL FORESTAL, 1999) indican que la tasa de pérdida de los terrenos agrícolas alcanzan un 1,3\% anual, lo cual es muy coincidente con el estudio actual.

Las praderas y matorrales presentaron una tasa de pérdida de 2,5\% anual, las causas principales se deben entre otras a los incentivos estatales, produciéndose una habilitación de superficie de este tipo de uso, hacia los terrenos agrícolas y otra parte importante habilitada directamente hacia la actividad forestal, atraídos los propietarios por los subsidios estatales (SANHUEZA; AZÓCAR, 2000), situación que permite además que los terrenos agrícolas también se transformen para el uso forestal. Estudios anteriores en la región (CORPORACIÓN NACIONAL FORESTAL, 1999), donde se consideró un periodo entre los años 1994 y 1998, indican una tasa de crecimiento anual del 1,3\%, esto se debe a que la intervención del bosque nativo es habilitada en grandes extensiones para uso de praderas y posteriormente pasan a plantaciones, situación similar ocurre con el bosque mixto.

\section{CONCLUSIONES}

El uso de las coberturas de ambos periodos permitió construir indicadores que explican el cambio de los diferentes usos del suelo, permitiendo revelar a nivel regional que ha existido una sobre-ocupación del territorio con plantaciones forestales con especies exóticas de pino y Eucalyptus, con una ocupación actual de 1.227 .788 ha y una tasa de incremento anual de $2,5 \%$, en desmedro de los usos praderas y mator- 
rales, terrenos agrícolas, bosque nativo, bosque mixto y humedales.

Respecto a la dinámica del cambio del uso del suelo que se produce a nivel provincial, se pudo determinar que las cuatro provincias mantienen las mismas conductas del cambio y coincidente con las observadas a nivel regional, es así, como en la provincia de Arauco el 52,3\% del territorio se está ocupado por plantaciones forestales con una tasa de incremento anual de $1,4 \%$, haciendo uso principalmente de áreas desprovistas de vegetación y terrenos agrícolas. Otro caso excepcional corresponde a la provincia de Concepción, con el porcentaje de uso del territorio y la tasa de incremento anual de $57,5 \%$ y $1,3 \%$ respectivamente.

Debido a la alta actividad de las empresas forestales en la región, se genera una fragmentación y degradación de los bosques nativos que están quedando y ocupación de terrenos agrícolas. Generando presiones a los pequeños propietarios en la reconversión en el uso del suelo con plantaciones exóticas de rápido crecimiento, maximizada por las bonificaciones que realiza el estado a este tipo de uso de la tierra.

\section{AGRADECIMIENTOS}

Los autores agradecen en forma especial al Laboratorio de Geomática de la Universidad Austral de Chile por permitir procesar la información de las coberturas vegetativas de la Región estudiada, también a la Corporación Nacional Forestal de Chile (CONAF) y a la Dirección de Investigación y Desarrollo (DID) de la Universidad Austral de Chile.

\section{REFERENCIAS}

AGUAYO, M. et al. Cambio del uso del suelo en el centro sur de Chile a fines del siglo XX. Entendiendo la dinámica espacial y temporal del paisaje. Revista Chilena de Historia Natural, Santiago, v. 82, n. 3, p. 361-374, 2009.

ALTAMIRANO, A.; LARA, A. Deforestación en ecosistemas templados de la precordillera andina del centro-sur de Chile. Revista Bosque, Valdivia, v. 31, n. 1, p. 53-64, 2010.

ALVES, D. S. Space-time dynamics of deforestation in Brazilian Amazonia. International Journal of Remote Sensing, Singapore, v. 23, n. 14, p. 2903-2908, 2002.

ARCGIS. Getting Started with ArcGIS. United States: [s. n.], 2001. 253 p.

AZÓCAR, G. et al. Urbanization patterns and their impacts on social restructuring of urban space in Chilean mid-cities: The case of Los Ángeles, central Chile. Land Use Policy, Adelaide, v. 24, n. 1, p. 199-21, 2007.

AZÓCAR, G.; SANHUEZA, R.; HENRÍQUEZ, C. Cambio en los patrones de crecimiento en una ciudad intermedia: el caso de Chillán en Chile Central. EURE, Santiago, v. 29, n. 87, p. 79-82, 2003.

CORPORACIÓN NACIONAL FORESTAL. Catastro y evaluación de recursos vegetacionales de Chile. Informe regional Octava Región. Santiago: Ministerio de Agricultura, 1999. 130 p.

DEZZEO, A. A.; SENDRA, J. B. Cambios ocurridos en la cobertura/uso de la tierra del parque nacional sierra de la culata. Mérida-Venezuela. Período 1988-2003. Geofocus: Revista Internacional de Ciencia y Tecnología de la Información Geográfica, Barcelona, v. 7, n. 8, 2008.

ECHEVERRÍA, C. et al. Rapid deforestation and fragmentation of Chilean temperate forests. Biological conservation, Montpelier, v. 130, n. 4, p. 481-494, 2006.

ECHEVERRIA, C. et al. Spatially explicit models to analyze forest loss and fragmentation between 1976 and 2020 in southern Chile. Ecological Modelling, Maryland, v. 212, n. 3, p. 439-449, 2008.

HENRÍQUEZ, C.; AZÓCAR, G.; ROMERO, H. Monitoring and modeling the urban growth of two midsized Chilean cities. Habitat International, California, v. 30, n. 4, p. 945-964, 2006.

INSTITUTO FORESTAL. Boletín estadístico: estadísticas forestales. Santiago: Ministerio de Agricultura, 2007. $163 \mathrm{p}$.

INSTITUTO FORESTAL. Boletín estadístico: estadísticas forestales. Santiago: Ministerio de Agricultura, 2008. $161 \mathrm{p}$.

INSTITUTO NACIONAL DE ESTADÍSTICAS. Boletín estadístico: estadísticas de Chile. Santiago: Ministerio de Economía, 2002. 204 p. 
LAMBIN, E. et al. Land use and land cover change implementation strategy, IGBP report, 48, IHDP, report 10. Estocolmo: [s. n.], 1999.

LAMBIN, E. F.; GEIST, H. J.; LEPERS, E. Dynamics of land-use and land-cover change in tropical regions. Annual review of environment and resources, California, v. 28, n. 1, p. 205-241, 2003.

LARA, A. et al. Componentes científicos clave para una política nacional sobre usos, servicios y conservación de los bosques nativos chilenos. Valdivia: Universidad Austral de Chile, Iniciativa Científica Milenio de Mideplan, 2003. 135 p.

LARA, A.; ECHEVERRÍA, C.; REYES, R. Bosques nativos. Estado del Medioambiente en Chile. Santiago: Universidad de Chile, 2002.

NAHUELHUAL, L. et al. Land-cover change to forest plantations: Proximate causes and implications for the landscape in south-central Chile. Landscape and Urban Planning, Michigan, v. 107, n. 1, p. 12-20, 2012.

PUYRAVAUD, J. P. Standardizing the calculation of the annual rate of deforestation. Forest Ecology and Management, Sydney, v. 177, n. 1, p. 593-596, 2003.

RUDEL, T. K. et al. Forest transitions: towards a global understanding of land use change. Global Environmental Change, Victoria, v. 15, n. 1, p. 23-31, 2005.

SALA, O. E. et al. Global biodiversity scenarios for the year 2100. Science, Washington, v. 287, n. 5459, p. 1770-1774, 2000.

SANDOVAL, V. Manual de Operaciones en Terreno, monitoreo del uso del suelo en Chile. Valdivia: Universidad Austral de Chile, Laboratorio de Geomática, 2008. 65 p.

SANDOVAL, V.; REAL, P. Modelamiento y prognosis estadística y cartográfica del cambio en el uso de la tierra. Revista Bosque, Valdivia, v. 26, n. 1, p. 55-63, 2005.

SANHUEZA, R.; AZÓCAR, G. Transformaciones ambientales provocadas por los cambios económicos de la segunda mitad del siglo XIX; provincia de Concepción. Revista Geográfica de Chile Terra Australis, Santiago, 45, p. 181-194, 2000.

SHEERAN, K. A. Forest conservation in the Philippines: a cost-effective approach to mitigating climate change? Ecological Economics, New Hampshire, v. 58, n. 2, p. 338-349, 2006.

TURNER, B. L.; LAMBIN, E. F.; REENBERG, A. The emergence of land change science for global environmental change and sustainability. Proceedings of the National Academy of Sciences, Washington, v. 104, n. 52, p. 20666-20671, 2007.

VERGARA G.; SANDOVAL, V. Manual del usuario del sistema de actualización del monitoreo nacional del uso del suelo en Chile. Valdivia: [s. n.], 2010. 55 p. 